\title{
Testing Pulsar Thermal Evolution Theories with Observation
}

\author{
Sachiko Tsuruta \\ Montana State University, Bozeman, MT 59717, USA
}

\begin{abstract}
With the successful launch of Chandra and XMM-Newton, the time has arrived when careful comparison of thermal evolution theories of isolated neutron stars with observations will offer a better hope for distinguishing among various competing neutron star cooling theories. For instance, the latest theoretical and observational developments may already exclude both nucleon and kaon direct Urca cooling. In this way we can now have a realistic hope for determining various important properties, such as the composition, superfluidity, the equation of state and stellar radius. These developments should help us obtain deeper insight into the properties of dense matter.
\end{abstract}

\section{Introduction}

The launch of the Einstein Observatory gave the first hope for detecting thermal radiation directly from the surface of neutron stars (NSs). However, the temperatures obtained by Einstein were only upper limits (e.g., Nomoto \& Tsuruta 1986). ROSAT offered the first confirmed detections (not just upper limits) for such surface thermal radiation from at least three cooling neutron stars, PSRs B0656+14, B0630+18 (Geminga) and B1055-52 (e.g., Becker 1995). Recently the prospect for measuring the surface temperature of isolated NSs, as well as obtaining better upper limits, has increased significantly, thanks to superior X-ray data from Chandra and from XMM-Newton, as well as data in lower energy bands from optical-UV telescopes such as the Hubble Space Telescope $(H S T)$. Consequently, the number of possible surface temperature detections has already increased to at least nine (see, e.g., Tsuruta et al. 2002, hereafter T02). Very recently Chandra offered an important upper limit to PSR J0205+6449 in 3C 58 (Slane, Helfand \& Murray 2002). More detections, as well as better upper limits, are expected from space missions planned for the immediate future. These developments have proved to offer serious "turning points" for the detectability of thermal radiation directly from the surface of isolated NSs. In addition, more careful and detailed theoretical investigation of various input microphysics has already started (see e.g., Lattimer, these proceedings; Takatsuka et al. 2001; Takatsuka et al. 2004, hereafter Ta04a; Tamagaki 2004, hereafter Ta04b). In this report we try to demonstrate that distinguishing among various competing NS cooling theories has started to become possible, by careful comparison of improved theories with new observations (see e.g., T02; Tsuruta et al. 2004, hereafter Ts04; Teter et al. 2004, hereafter Te04). 


\section{Neutron Star Cooling Theories}

The first detailed cooling calculations (Tsuruta \& Cameron 1966) showed that isolated NSs can be warm enough to be observable as X-ray sources for about a million years. After the supernova explosion, a newly formed NS first cools via various neutrino emission mechanisms before the surface photon radiation takes over. Among the important factors which seriously affect the nature of NS cooling are: neutrino emission processes, superfluidity of constituent particles, composition, mass, and the equation of state (EOS). In this paper, for convenience, the conventional, slower neutrino cooling mechanisms, such as the modified Urca, plasmon neutrino and bremsstrahlung processes, will be called "standard cooling". On the other hand, the more "exotic" extremely fast cooling processes, such as the direct Urca processes involving nucleons, hyperons, pions, kaons, and quarks, will be called "non-standard" processes (see e.g., Tsuruta 1998, hereafter T98; Tsuruta \& Teter 2001, hereafter TT01).

The composition of the NS interior is predominantly neutrons with only a small fraction of protons and electrons (and possibly muons also) when the interior density is not high (the central density $\rho^{c}<10^{15} \mathrm{~g} \mathrm{~cm}^{-3}$ ). For higher densities, more "exotic" particles, such as hyperons, pions, kaons and quarks, may dominate the central core. Therefore, when the star is less massive and hence less dense, it will be a neutron star with the interior consisting predominantly of neutrons (no "exotic" particles) and it will cool with the slower, "standard" neutrino processes. On the other hand when $\rho^{c}$ exceeds the transition density, $\rho_{t r}$, to exotic matter, the transition from nucleons to "exotic" particles takes place. Therefore, more massive stars, for which $\rho^{c}$ exceeds $\rho_{t r}$, possess a central core consisting of exotic particles. In that case, the non-standard fast cooling takes over. ${ }^{1}$ The observational data suggest that there are at least two classes of NS, hotter ones and cooler ones. The most natural explanation is that hotter stars cool by the standard, slower cooling processes, while the cooler ones cool by one of the fast non-standard processes. This interpretation follows naturally from the effect of slightly different masses, with hotter stars somewhat less massive than cooler ones (see $\S 3$ for details).

As the central collapsed star cools after a supernova explosion and the interior temperature falls below the superfluid critical temperature, $T^{c r}$, some constituent particles become superfluid. That causes exponential suppression of both specific heat (and hence the internal energy) and all neutrino processes involving these particles. The net effect is that the star cools more slowly (and hence the surface temperature and luminosity will be higher) during the neutrino cooling era due to the suppression of neutrino emissivity. Therefore, we point out that non-standard fast cooling will be no longer so fast if the superfluid energy gap, which is proportional to $T^{c r}$, is significant. In addition to various neutrino cooling mechanisms conventionally adopted in earlier calculations, recently the

\footnotetext{
${ }^{1}$ Note, however, that if proton fraction in the neutron matter is exceptionally high, i.e. $>15 \%$, non-standard fast cooling can take place in a NS without exotic particles, through the nucleon direct Urca process. This can happen for certain types of EOS models which allow such high proton concentration above a certain critical density (Lattimer et al. 1991). In order to include this option, in the following discussion we will call a fast non-standard process an "exotic process" rather than "a process involving exotic particles".
} 
"Cooper pair neutrino emission" (Flowers, Ruderman \& Sutherland 1976) was "rediscovered" to be also important under certain circumstances. This process takes place when the participating particles become superfluid, and the net effect is to enhance, for some superfluid models, the neutrino emission right after the superfluidity sets in.

\section{Most Recent NS Thermal Evolution Models and Comparison with New Observations}

\subsection{Latest Thermal Evolution Models}

We calculated NS thermal evolution ${ }^{2}$ adopting the most up-to-date microphysical input and a fully general relativistic, "exact" evolutionary code (i.e., without making isothermal approximations). This code was originally constructed by Nomoto \& Tsuruta (1987), and has been continuously updated. Our input neutrino emissivity consists of all possible mechanisms, including Cooper pair emission, both in the stellar core and crust. The vortex creep heating is also included, unless otherwise stated. See Ts04, Te04 for the details. The results are summarized in Figure 1.

Figure 1(a) compares thermal evolution of a neutron star and stars with a pion core. The EOS adopted is the "TNI3U Model" recently constructed by Ta04a, which is somewhat stiffer than medium. ${ }^{3}$ This EOS refers to pion condensates when density exceeds $\rho_{t r}^{\pi}$, the density where transition from neutron matter to pion matter takes place, which is set to be $4 \rho_{0}$ (where $\rho_{0}=2.8 \mathrm{x}$ $10^{14} \mathrm{~g} \mathrm{~cm}^{-3}$ is the nuclear density). Then, for this particular EOS the central density is $\rho^{c}=\rho_{t r}^{\pi}$ for a $1.5 \mathrm{M}_{\odot}$ star. ${ }^{4}$ The solid curve refers to thermal evolution (including heating) of a $1.4 \mathrm{M}_{\odot}$ star. Since for this star $\rho^{c}<\rho_{t r}^{\pi}$, it consists predominantly of neutrons with only several percent protons, and it cools by slower "standard" processes. For the core superfluid model for neutrons we adopt the OPEG-B Model recently constructed by Ta04b for neutron matter. For proton superfluidity we adopt the model by Chao, Clark \& Yang (1972). The Cooper pair neutrino emissivity derived by Yakovlev, Levenfish \& Shibanov (1999) is adopted for both neutrons and protons in the central core and neutrons in the inner crustal layers. For the vortex creep heating we adopt the model with heating parameter $K=10^{37}$ ergs $\mathrm{m}^{-3 / 2} \mathrm{~s}^{2}$ and magnetic field $B=10^{12} \mathrm{G}$ (Umeda, Tsuruta \& Nomoto 1994, hereafter UTN94). The long dashed and dashed curves present thermal evolution of $1.6 \mathrm{M}_{\odot}$ and $1.7 \mathrm{M}_{\odot}$ stars, respectively. These stars cool predominantly by the non-standard pion direct Urca process, because for these more massive stars $\rho^{c}>\rho_{t r}^{\pi}$ and hence the central core consists of pion condensates. For the superfluid model for the pion-condensed phase, we adopt a medium superfluid gap model for pion condensates, called the E1-0.6 Model

\footnotetext{
${ }^{2}$ We adopt the expression "thermal evolution" when we include not only cooling but also heating.

${ }^{3}$ Often an EOS is referred to as "stiff" when the consequent stellar model is more extended and hence less dense, while it is referred to as "soft" if it is more compact and denser.

${ }^{4}$ Note that very recent observations suggest that mass of an isolated neutron star may be somewhat higher than $1.4 \mathrm{M}_{\odot}$ (see $\S 3.2$ ).
} 

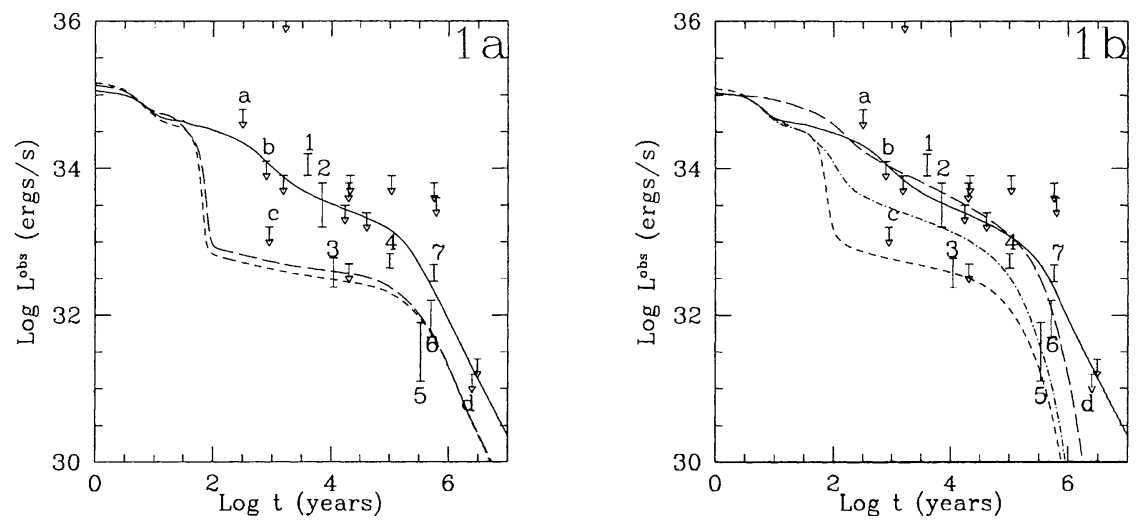

Figure 1. Thermal evolution curves with the newest observational data. The surface photon luminosity, $L^{\text {obs }}$, which corresponds to surface temperature (both observed at infinity) is shown as a function of age; see the text for the details. The vertical bars refer to confirmed and possible temperature detection data with error bars, for (1) RX J08224300, (2) 1E 1207.4-5209, (3) the Vela pulsar, (4) PSR B0656+14, (5) Geminga, (6) RX J1856-3754, and (7) PSR B1055-52. (In spite of possible positive detections, the sources RX J0002+6246, and RX J0720.43125 are not shown because currently there are still some uncertainties associated with their age estimates.) The downward arrows refer to upper limits. Some interesting objects are: (a) Cas A point source, (b) Crab pulsar, (c) PSR J0205+6449 in 3C 58, and (d) PSR B1929+10. See Te04 and Ts04 for references and further details on the data.

(Umeda et al. 1994, hereafter U94; Takatsuka \& Tamagaki 1982). See Te04 for the details.

In Figure 1(b), hyperon cooling is compared with standard thermal evolution curves. The critical transition density from neutron matter to hyperon matter, $\rho_{t r}^{Y}$, is set at $4 \rho_{0}$. For $\rho>\rho_{t r}^{Y}$, we adopt the TNI3U EOS for hyperon matter recently calculated by Ta04a. Other input microphysical parameters are the same as in Figure 1(a). The solid curve refers to thermal evolution (heating included) of a $1.4 \mathrm{M}_{\odot} \mathrm{NS}$ with the standard scenario. The dashed curve presents cooling of a $1.6 \mathrm{M}_{\odot}$ hyperon star. For the TNI3U EOS adopted, we find that $\rho^{c}$ $=\rho_{t r}^{Y}$ for a $1.5 \mathrm{M}_{\odot}$ star. Therefore, our $1.6 \mathrm{M}_{\odot}$ star contains a hyperon core and hence the predominant cooling mechanism is the non-standard hyperon direct Urca process. As the superfluid model for hyperons we adopt Ehime Model for hyperon matter (Ta04a). See Ts04 for further details.

Figure 1(b) also shows two more curves, dot-dashed and long dashed, for the standard cooling of $1.4 \mathrm{M}_{\odot}$ NSs to explore the effect of heating and core neutron superfluidity. In the dot-dashed curve, all input parameters are the 
same as for the solid curve, except that heating is switched off. Therefore, by comparing the dot-dashed curve with the solid curve, we can see the effect of heating, which we note to be significant. Except that core neutron superfluidity is not included, the input parameters to the long dashed curve are the same as for the dot-dashed curve.

\subsection{Comparison with Observation}

In Figure 1, thermal evolution curves are compared with the latest observational data. The numbers refer to either confirmed or possible detections while letters refer to some interesting upper limits. We may note that the data suggest the existence of at least two classes of sources, hotter stars, e.g. (1) RX J08224300, (2) 1E 1207.4-5209 and (7) PSR B1055-52, and cooler stars, e.g. (c) PSR J0205+6449, (3) the Vela pulsar and (5) Geminga. The hotter sources are consistent with the solid curves, the standard cooling of a $1.4 \mathrm{M}_{\odot} \mathrm{NS}$ when heating is included. The source (7) is slightly above the solid curves, but that is easily explained when the age uncertainty, of at least a factor of $\sim 2$ or larger, is taken into account. The source (1) is somewhat higher than the solid curves, but that is easily explained by, e.g. the presence of magnetic envelopes with light elements (e.g., Potenkin et al. 2003). Comparison of cooler star data with pion and hyperon curves confirms the earlier conclusion (e.g., T98; TT01; T02) that non-standard cooling of a more massive star is required for these cooler data. In that case, we find that significant superfluid suppression is required if the cooler data are detections. The age uncertainty should not affect this conclusion, especially for younger cooler sources such as (c) and (3), because the slope of the curves in these younger years is small. The data point for (4), PSR $\mathrm{B} 0656+14$, lies between the $1.4 \mathrm{M}_{\odot}$ and $1.6 \mathrm{M}_{\odot}$ curves, which implies its mass is between these masses in our current model. The conclusion is that all of the observed data are most naturally and consistently explained as the effects of stellar mass, superfluidity of the constituent particles, and heating.

At least for binary pulsars, observations offer stringent constraints on the mass of a NS, to be very close to $1.4 \mathrm{M}_{\odot}$ (e.g., Brown, Weingartner \& Wijers 1996). If this evidence extends to isolated NSs also, then the EOS should be such that the mass of the star whose central density is very close to the transition density (where the non-standard process sets in) should be very close to $1.4 \mathrm{M}_{\odot}$. With the EOS of medium stiffness, our earlier work (T98; TT01; T02) finds that this transition takes place for stellar masses near $1.4 \mathrm{M}_{\odot}$. In the current models presented here, we take account of the most recent report that some new observations suggest the mass of isolated NSs to be somewhat higher (see Nice, Splaver \& Stairs, these proceedings). In that case, the EOS has to be stiffer than medium. This is because a stiffer EOS corresponds to a larger mass for a given central density. That is why our current model adopts an EOS stiffer than medium. In conclusion it may be emphasized that in this way comparison of thermal evolution curves with observed temperature data has the potential for determining the EOS and hence the radius, if the mass is fixed. For instance, comparison of this kind may already eliminate very soft and very stiff EOSs.

The qualitative behavior of all non-standard scenarios is similar if their transition density is the same (see e.g., UTN94; T98). However, here we try to demonstrate that it is still possible to offer comprehensive assessment of at least 
which options are more likely while which are less likely. First of all, we note that all of the non-standard mechanisms are too fast to be consistent with any observed detection data, even with heating (see e.g., UTN94; T98). That means significant suppression of neutrino emissivity due to superfluidity is required, if the cooler data are detections. However, Takatsuka \& Tamagaki (1997) already showed, through careful microphysical calculations, that for neutron matter with such high proton concentration as to permit the nucleon direct Urca process, the superfluid critical temperature, $T^{c r}$, should be extremely low, $\sim$ several $\times 10^{7}$ $\mathrm{K}$, not only for neutrons but also for protons. Here we emphasize that this conclusion does not depend on the nuclear models adopted for the calculations. On the other hand, the observed NSs, which are to be compared with cooling curves, are all hotter (the core temperature being typically $\sim 10^{8} \mathrm{~K}$ to several times $10^{8} \mathrm{~K}$ ). That means the core particles are not yet in the superfluid state in these observable NSs. The conclusion is that a star cooling with nucleon direct Urca would be too cold to be consistent with the detection data. The same argument applies to the kaon cooling also (Takatsuka \& Tamagaki 1995). Further details are found in TT01, T02, Ts04 and Te04. As for the hyperon option, Figure 1(b) shows that it can be a viable possibility if the Ehime Model adopted is valid. However, recently the Gifu-Kyoto nuclear experimental group (Takahashi et al. 2001) reports that the superfluid gap for hyperons would be much smaller. If so, hyperon cooling also would be in trouble with the same reason as for nucleon direct Urca if the cooler data are detections, due to lack of superfluid suppression. The problem for quarks is that theoretically there are still too many unknown factors to offer the level of exploration possible for the other options (see Ts04). On the other hand, detailed theoretical investigations have already shown that $T^{c r}$ for pion condensates is realistically high and hence pions will safely be in a superfluid state. The conclusion is that pion cooling is consistent with both theory and observation. See T98, TT01, T02, Ts04 and Te04 for further details.

\section{NS Cooling Models by Other Groups}

Various other groups have calculated neutron star thermal evolution. A comprehensive review is found in, e.g., T98. Due to lack of space here we comment on only the latest work by Yakovlev's group, e.g., Yakovlev et al. (2004). Although very often these authors adopted simplified "toy models" with isothermal and other various approximations, their results and ours generally agree, at least qualitatively, when similar input is applied. ${ }^{5}$ There are, however, some serious differences in our interpretation of the results. For instance, since in the presence of core superfluid neutrons, standard cooling is not hot enough for the data of PSR B1055-52, these authors conclude that neutron superfluidity must be so weak as to be negligible. However, this conclusion contradicts with the results of serious microphysical calculations of neutron superfluidity (see Ta02b, T02, Ts04 for the details), which find that neutron superfluidity should not be

\footnotetext{
${ }^{5}$ Yakovlev \& Haensel (2003) state that the observational data of T02 are wrong. However, this is due to a mistake on their part in converting surface temperature to luminosity. Also they misunderstood some of the models presented in T02. See Ts04 for the details.
} 
so weak for normal neutron matter with a small proton concentration where standard cooling operates. On the other hand, we have shown (see Fig. 1) that this discrepancy disappears when heating is included properly in calculations of thermal evolution of isolated (not binary) neutron stars - and hence no contradiction with theories. Furthermore, for detailed comparison of non-standard cooling with the cooler star data, these authors chose the nucleon direct Urca process, which they called Durca. To be consistent with the apparent need for no (or only very weak) neutron superfluidity for slower standard cooling to explain the hot data, these authors conclude that observational data require strong proton superfluidity to supply sufficient superfluid suppression, in order to explain the cooler detection data by Durca. However, it was already emphasized in $§ 3.2$ that theoretically, proton superfluidity must be so weak that its suppression effect should be negligible for models which will allow Durca. These authors adopted the proton superfluid model for normal neutron matter with a small proton fraction, which fails to apply when the proton concentration is high enough so that Durca can operate.

\section{Concluding Remarks}

We have shown that the most up-to-date observed temperature data are consistent with the current thermal evolution theories of isolated NSs if less massive stars cool by standard cooling while more massive stars cool with non-standard cooling, and if heating is also in operation. Among various non-standard cooling scenarios, both Durca and kaon cooling should be excluded if the cooler data are detections. The major reason is that for Durca to be operative, a high proton concentration is required, which weakens superfluidity of both protons and neutrons. A similar argument applies to kaon cooling. Hyperon cooling may be in trouble if the cooler data are detections and if the hyperon superfluid gap should be as small as reported by recent nuclear experiments. On the other hand, pion cooling is still consistent with both observation and theory. The important conclusion is that if the cooler data are detections, the presence of "exotic" particles, most likely pion condensates, is required within a very dense star.

The capability of constraining the composition of NS interior matter purely through observation alone will be limited, and hence it will be very important to exhaust all theoretical resources. Theoretical uncertainties are also very large, especially in the supranuclear density regime. However, here we emphasize that we should still be able to set acceptable ranges of theoretical feasibility, at least to separate more likely from less likely models. More and better data expected soon from Chandra, XMM-Newton, HST and future third generation missions, when combined with improved theories, should give still better insight into some fundamental problems in dense matter physics.

Acknowledgments. I acknowledge the contributions by my collaborators, W. Chandler, M. A. Teter, T. Takatsuka, R. Tamagaki, K. Fukumura, G. Pavlov, K. Nomoto, T. Tatsumi and H. Umeda, to the results presented in this paper. Our work for this paper has been supported in part by NASA grants NAG5-3159, NAG5-12079, AR3-4004A and G02-3097X. 


\section{References}

Becker, W. 1995, PhD thesis, Ludwig-Maximilians-University München Brown, G. E., Weingartner, J. C., \& Wijers, R. A. 1996, ApJ, 463, 297 Chao, N. C., Clark, J. W., \& Yang, C. H. 1972, Nucl. Phys. A, 179, 320 Flowers, E. G., Ruderman, M., \& Sutherland, P. G. 1976, ApJ, 205, 541 Lattimer, J. M., Pethick, C. J., Prakash, M., \& Haensel, P. 1991, Phys. Rev. Lett., 66, 2701

Nomoto, K., \& Tsuruta, S. 1986, ApJ, 305, L19

- 1987, ApJ, 312, 711

Potenkin, A. Y., Yakovlev, D. G., Chabrier, G., \& Gnedin, O. Y. 2003, ApJ, 594,404

Slane, P. O., Helfand. D. J., \& Murray, S. S. 2002, ApJ, 571, L45

Takahashi, H. et al. 2001, Phys. Rev. Lett., 87, 21

Takatsuka, T., \& Tamagaki, R. 1982, Prog. Theor. Phys., 67, 1649

- 1995, Prog. Theor. Phys., 94, 457

- 1997, Prog. Theor. Phys., 97, 345

Takatsuka, T., Nishizaki, S., Yamamoto, Y., \& Tamagaki, R. 2001, Nucl. Phys. A, 691, 254c

-2004, in preparation (Ta04a)

Tamagaki, R. 2004, in preparation (Ta04b)

Teter, M.A., Candler, W., Tsuruta, S., Takatsuka, T., Tamagaki, R., Fukumura, G. Pavlov, K., Nomoto, K., Umeda, H., \& Tatsumi,T. 2004, in preparation ( $\mathrm{Te} 04)$

Tsuruta, S. 1998, Phys. Rep., 292, 1 (T98).

Tsuruta, S., \& Cameron, A. G. W. 1966, Canad. J. Phys., 44, 1863

Tsuruta, S., \& Teter, M. A. 2001 in Proceedings of the 20th Texas Symposium, ed. H. Martel \& J.C. Wheeler (AIP), 507 (TT01)

Tsuruta, S., Teter, M. A., Takatsuka, T., Tatsumi, T., Tamagaki, R. 2002, ApJ, 571, L143 (T02)

Tsuruta, S., Chandler, W., Teter, M. A.,Takatsuka, T., Tamagaki, R., Fukumura, G. Pavlov, K., Nomoto, K., Umeda, H., \& Tatsumi,T. 2004, in preparation ( $\mathrm{Ts} 04)$

Umeda, H., Nomoto, K., Tsuruta, S., Muto, T., \& Tatsumi, T. 1994, ApJ, 431, 309 (U94)

Umeda, H., Tsuruta, S., \& Nomoto, K. 1994, ApJ, 433,256 (UTN94)

Yakovlev, D. G., Gnedin, O. Y., Kaminker, A. D., Levenfish, K. P., \& Potenkin, A. Y. 2004, Adv. Sp. Res., 33, 523

Yakovlev, D. G., Levenfish, K. P., \& Shibanov, Y. A. 1999, Phys. Usp., 42, 737

Yakovlev, D. G. \& Haensel, P. 2003, A\&A, 407, 259 